\title{
Dp71 depleted HBE cells displayed increased DNA damage and apoptosis induced by $\mathrm{H}_{2} \mathrm{O}_{2}$
}

Sichuang $\operatorname{Tan}^{2}$, Shuai Zhao ${ }^{2,6}$, Xuefei Xiao ${ }^{5}$, Lan Xiao ${ }^{4}$, Jinliang Xie ${ }^{3}$ and Sipin $\operatorname{Tan}^{1 *}$ (D)

\author{
* Correspondence: springtan@csu. \\ edu.cn \\ ${ }^{1}$ Key Laboratory of Sepsis \\ Translational Medicine of Hunan, \\ Department of Pathophysiology, \\ Xiangya School of Medicine, Central \\ South University, Changsha, Hunan \\ Province 410008, People's Republic \\ of China \\ Full list of author information is \\ available at the end of the article
}

\begin{abstract}
Human bronchial epithelium (HBE)-Dp71 anti-sense(AS)cells with stably transfected Dp71 siRNA plasmids were prepared for further exploration of Dp71 biological traits in cells other than PC12. HBE-Dp71AS cells displayed increased DNA damage induced by $\mathrm{H}_{2} \mathrm{O}_{2}$. Apoptosis of HBE-Dp71AS cells induced by $\mathrm{H}_{2} \mathrm{O}_{2}$ was increased via enhancing caspase 3, caspase 8 and caspase 9. HBE-Dp71AS cells also displayed decreased proliferation and clonogenic formation. RAD51 was proved to be a new binding partner of Dp71 by co-immunoprecipitation (Ip) and immunofluorescence. Reduced RAD51 mRNA and protein levels were observed in HBE-Dp71AS cells. Decreased lamin B1, focal adhesion kinase (FAK), phosphorylated focal adhesion kinase ( $p$-FAK) and phosphorylated protein kinase $B$ ( $p$-AKT) were detected in the HBE-Dp71AS cells, which functioned together with RAD51 as the molecular explanations for the character alterations of HBE-Dp71AS cells.
\end{abstract}

Keywords: Dp71, DNA damage, Apoptosis, RAD51, FAK

\section{Introduction}

Dystrophin Dp71 is one of the most widely expressed isoforms of dystrophin, the pathogenic gene of Duchenne muscular dystrophy (DMD), an X-linked recessive disorder [1]. Functioning as one of the most ubiquitously expressed isoforms of dystrophin, Dp71 is a 70- to $75-\mathrm{kDa}$ protein located in all tissues except skeletal muscle $[2,3]$. Previous research on Dp71 identified its crucial role for cell adhesion, neuronal differentiation and the cell cycle in PC12 cells. Dp71 was proved to be a putative tumor suppressive gene in gastric cancer [4-6]. Our preliminary clinical work also identified reduced Dp71 expression in lung cancer. Considering HBE as a usual cell model for pulmonary functional analysis, a shRNA strategy was used to knock down Dp71 in HBE to further clarify its biological significance. HBE-AS cells displayed increased DNA damage under oxidative stress, and decreased proliferation and clone formation capabilities. In a caspase-dependent way, HBE-AS cells displayed an increased apoptosis rate induced by $\mathrm{H}_{2} \mathrm{O}_{2}$. Our further characterization of HBE-AS cells identified RAD51, lamin B1, FAK and AKT to be the molecular explanations for the altered phenotypes of HBE-AS cells.

(c) The Author(s). 2019 Open Access This article is distributed under the terms of the Creative Commons Attribution 4.0 International License (http://creativecommons.org/licenses/by/4.0/), which permits unrestricted use, distribution, and reproduction in any medium, provided you give appropriate credit to the original author(s) and the source, provide a link to the Creative Commons license, and indicate if changes were made. The Creative Commons Public Domain Dedication waiver (http://creativecommons.org/ publicdomain/zero/1.0/) applies to the data made available in this article, unless otherwise stated. 


\section{Material and methods \\ Construction of Dp71 short hairpin RNA plasmid}

According to the open reading frame of the human Dp71 gene (NM_004015), one siRNA sequence $\left(5^{\prime}\right.$-gcactttaattatgacatc- $\left.3^{\prime}\right)$ was selected. The scrambled sequence $\left(5^{\prime}\right.$ ttctccgaacgtgtcacgt- $\left.3^{\prime}\right)$ which has no significant homology with human gene sequences was included as a negative control. Two complementary oligonucleotides for Dp71 (5' gatcccgtctttagctgacctgaataactcgagttattcaggtcagctaaagactttttggat-3' and $5^{\prime}$-agctatccaaa aagtctttagctgacctgaataactcgagttattcaggtcagctaaagacgg- $\left.3^{\prime}\right)$, and for the negative control (5' -gatcccttctccgaacgtgtcacgtctcgagacgtgacacgttcggagaattttggat-3' and $5^{\prime}$-agctatc caaaaattctccgaacgtgtcacgtctcgagacgtgacacgttcggagaagg-3'), were synthesized by Invitrogen. Sense or antisense strands are in bold letters and stem loop sequences are in italics. They were annealed to generate double-stranded DNAs and ligated into the linearized shRNA (short hairpin RNA) eukaryotic expression vectors purchased from Genechem (Shanghai, China, containing hU6-MCS-CMV-GFP-SV40-Neomycin elements) to construct Dp71 shRNA or control empty shRNA vectors, which were termed Dp71AS and Dp71 empty shRNA vector (E) respectively. The nucleotide sequences of the plasmids were verified by automated DNA sequencing.

\section{Cell culture and generation of stable transfectants}

HBE was obtained from the Culture Center, Chinese Academy of Medical Sciences (Shanghai, China). HBE cells were cultured in the same condition as described previously [7]. For stable transfectants, $5 \mu \mathrm{g}$ of Dp71shRNA plasmid or $5 \mu \mathrm{g}$ of control empty shRNA plasmid was mixed with $15 \mu \mathrm{l}$ of Lipofectamine in serum- and antibiotics-free 1640, and the DNA/Lipofectamine mixture was added to the cell culture medium and incubated in the incubator for $4 \mathrm{~h}$. The transfection mixture was removed and cells were maintained in 1640 supplemented with sera. Selection of stable transfectants was initiated with $600 \mu \mathrm{g} / \mathrm{ml}$ of G418 (Invitrogen) $48 \mathrm{~h}$ after transfection, a neomycin analog. The stable transfected HBE cells were named HBE-DP71AS and HBE-Dp71E respectively.

\section{Isolation of cell extracts and western blot analysis}

Cultured cells were collected by centrifugation at $1200 \mathrm{rpm}$ for $5 \mathrm{~min}$, and washed twice with PBS. Protein extraction, concentration determination, 10\% SDS-PAGE electrophoresis, and membrane incubation with the corresponding primary antibody (rabbit anti-dystrophin, rabbit anti-RAD51 polyclonal antibody purchased from Abcam; rabbit anti-FAK polyclonal antibody, p-FAK polyclonal antibody; rabbit anti-Akt polyclonal antibody, p-Akt polyclonal antibody; rabbit anti-phospho-histone H2AX ( $\gamma \mathrm{H} 2 \mathrm{AX}$; Ser 139) antibody (Bioworld Technology, Inc) was performed as described previously. After three washes with TBS-T, horseradish peroxidase-conjugated antirabbit IgG was used as the secondary antibody and developed using the ECL Western blotting analysis system (Amersham-Pharmacia).

\section{Quantitative real-time polymerase chain reaction (QRT-PCR) and RT-PCR}

The following primers were used and they produced a $157 \mathrm{bp}$ PCR product for Dp71: $173 \mathrm{bp}$ PCR product for FAK, $146 \mathrm{bp}$ PCR product for lamin B1, $160 \mathrm{bp}$ PCR products 
for RAD51 and $181 \mathrm{bp}$ PCR product for $18 \mathrm{~s}$. The primers are: LMNB1 (Human Accession NM_005573) F:5'tccaggagaaggaggagctg3', R:5'ggtctcgtagagcgccttg3'; Dp71 (Human Accession NM_004017.2) F:5'ttggcagtcaaacttcggactc3',R:5'gtgtcctctctcattggctttc cag3'; FAK (Human Accession L13616.1) F: 5' tccccagagctcctcaagaa 3',R: 5' tactcgctccattgcaccag3'; RAD51 (Human Accession D14134.1) F: 5' gggaagacccagatctgtca 3', R: 5' catcactgccagagagacca 3'; Human 18S (NM_022551) F: 5' aaatagcctttgccatcactgcc3',R: $5^{\prime}$ gttcaagaaccagtctgggatc $3^{\prime}$.

\section{Cell viability assay}

The cell viability was assessed by conducting the 3-(4,5-dimethylthiazol-2-yl)-2,5-diphenyltetrazolium bromide (MTT) assay. MTT assay and results interpretations were performed as described previously [8].

\section{Plate colony formation assay}

Clone formation assay was performed as described previously. Clone formation efficiency was calculated according to the formula: (clone number/plated cell number) $\times$ $100 \%[7]$.

\section{Apoptosis assay}

Apoptosis of HBE, HBE-Dp71AS and HBE-Dp71E cells in the log growth phase were induced by $0.2 \mathrm{mM} \mathrm{H}_{2} \mathrm{O}_{2}$ (Sigma, St. Louis, USA) for $16 \mathrm{~h}$. The cells then were harvested by trypsinization for flow cytometry. Apoptosis was quantified using the PE Annexin V apoptosis detection kit (BD Pharmingen, San Diego, USA) according to the manufacturer's protocol. Cell analyses were made using a FACSCalibur flow cytometer (Becton-Dickinson, Mountain View, CA) and CellQuest software (BD Biosciences). Each assay was repeated 3 times [7].

\section{Measurement of caspase 3, 8, 9 activities}

The caspase fluorescent assay kits specific for caspase 3, caspase 8 and caspase 9 (BioVision, San Francisco, USA) were used to detect caspase activation by measuring the cleavage of a synthetic fluorescent substrate. Cell treatment and fold increases in caspase 3, caspase 8 and caspase 9 activities were determined as described previously [8].

\section{Alkaline comet assay for DNA damage}

To perform the comet assay, the cell suspension of each cell group was mixed with low melting-point agarose at $37^{\circ} \mathrm{C}$, to a final concentration of $0.7 \%$. The mixture $(15 \mu \mathrm{l})$ was pipetted onto slides pretreated with $0.5 \%$ normal-melting-point agarose, to retain the agarose cell suspension. The drop containing the cells was covered with a glass cover slip $(24 \mathrm{~mm} \times 24 \mathrm{~mm})$ and left at $4{ }^{\circ} \mathrm{C}$ for $5 \mathrm{~min}$. The cover slips were gently removed and the slides were then ready for processing. The alkaline comet assay was performed using the basic rationale of Singh et al. The slides were then incubated in the dark for $30 \mathrm{~min}$ in cold electrophoresis buffer $(300 \mathrm{mM}$ $\mathrm{NaOH}, 1 \mathrm{mM}$ EDTA, 1\% (v/v) DMSO, pH 13) to allow the DNA to unwind before electrophoresis at $25 \mathrm{~V}$ for $25 \mathrm{~min}$. After neutralization with $0.5 \mathrm{M}$ Tris- $\mathrm{HCl}(\mathrm{pH}$ 8.0), the slides were stained with $50 \mu \mathrm{l}$ ethidium bromide $(30 \mu \mathrm{g} / \mathrm{mL}$, Absin 
Bioscience Inc., China). Finally, the images were taken by fluorescence microscope and at least 120 randomly selected cells (30 cells from each of the three replicate slides) were analyzed per sample and analyzed using the Comet Assay Software Pect (CASP 1.2.3 beta 1) (http://casplab.com/download). Parameters of tail moment (\% DNA in tail $\times$ tail length), tail length, and percent of DNA in tail, the most frequently used parameters in the comet assay, were used in this study.

\section{Immunofluorescence and confocal microscopy analysis}

The immunofluorescence and confocal microscopy analysis of Dp71, Rad51 and $\gamma$ $\mathrm{H} 2 \mathrm{AX}$ in HBE was as follows: After the three HBE cells were cultured on glass coverslips for $24 \mathrm{~h}$, cells were treated with $200 \mu \mathrm{M} \mathrm{H} \mathrm{H}_{2} \mathrm{O}_{2}$ for $30 \mathrm{~min}$ as described previously, treated cells and untreated cells were incubated overnight at $4{ }^{\circ} \mathrm{C}$ with the primary anti-dystrophin, anti-RAD51 and anti- $\gamma-\mathrm{H} 2 \mathrm{AX}$ antibody. Cells were incubated for 10 $\mathrm{min}$ at $37^{\circ} \mathrm{C}$ with $1 \mathrm{mg} / \mathrm{ml}$ 49,6-diamidino-2-phenylindole (DAPI) for counterstaining, After washing, coverslips were mounted on microscope slides with VectaShield (Vector Laboratories, Inc., Burlingame, CA, USA) and analyzed in a confocal and multiphoton microscope (TCS-SP5, Leica Microsystems, Heidelberg, Germany), using an oil immersion 636 objective. Co-localization of FITC, TRITC, and DAPI staining was analyzed in single optical sections obtained for two channels throughout the $\mathrm{Z}$ axis.

\section{Immunoprecipitation}

Total protein extracts in a final volume of $250 \mathrm{ml}$ were incubated overnight at $4{ }^{\circ} \mathrm{C}$ with $5 \mu \mathrm{g}$ of rabbit anti-lamin B1, $5 \mu \mathrm{g}$ of rabbit anti-Dp71 antibody, $5 \mu \mathrm{g}$ of rabbit anti-FAK, and $5 \mu \mathrm{g}$ of rabbit anti-RAD51 antibody, previously bound to protein G magnetic beads (Millipore). An irrelevant rabbit polyclonal antibody bound to protein G magnetic beads was performed as a negative control. The immune complexes were precipitated by placing the tube into the magnetic stand (Millipore) and washing 3 times with $500 \mu \mathrm{l}$ of PBS containing 0.1\% Tween 20. Precipitated proteins were separated by SDS-PAGE and analyzed by Western blotting with mouse anti-lamin B1, mouse anti-Dp71 antibody, mouse anti-RAD51 and mouse anti-FAK antibody.

\section{Statistical analyses}

All assays were repeated 3 times to ensure reproducibility. Results were displayed as mean \pm SE. One-way ANOVA and LSD were used to analyze all experimental data. All statistical analyses were performed with SPSS software (version 17.0; SPSS Inc., Chicago, IL, USA). $P<0.05$ was considered as indicating a statistically significant difference.

\section{Results}

\section{Establishment of Dp71 depleted HBE cell lines}

After 4 weeks' selection, G418-resistant HBE cells were obtained. According to the short hairpin RNA plasmids transfected, the cells were named HBE-Dp71AS and HBE-Dp71E. Western blotting showed that the protein expression of Dp71 was markedly downregulated by $70 \%$ in cells transfected with the Dp71 shRNA 


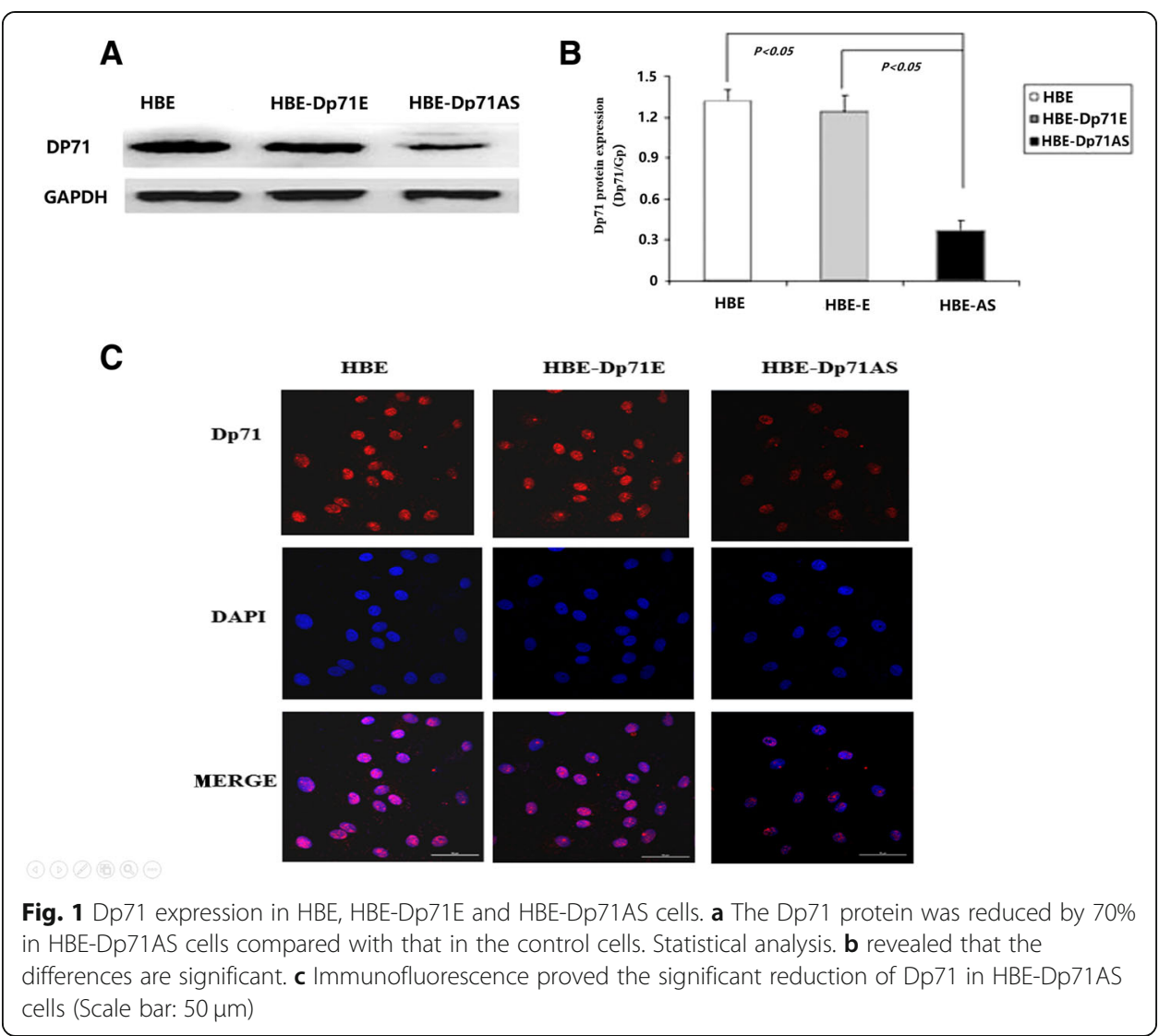

construct (Fig. 1a), while transfection of the control empty vector had little effect on Dp71 expression. These results suggested that Dp71 shRNA constructs potently and specifically inhibited endogenous Dp71 protein expression in HBE cells, and the differences were statistically significant (Fig. 1b). Immunofluorescence also clearly showed reduced Dp71 expression in HBE-Dp71AS cells (Fig. 1c). The stable HBE-Dp71AS and HBE-Dp71E cell lines were used for further functional analyses.

\section{HBE-Dp71 AS cells displayed increased DNA damage induced by $\mathrm{H}_{2} \mathrm{O}_{2}$}

After the HBE, HBE-Dp71E and HBE-Dp71AS cells had been exposed to $0.2 \mathrm{mM} \mathrm{H}_{2} \mathrm{O}_{2}$ for $30 \mathrm{~min}$, the comet assay was used to analyze single strand breaks in these three HBE cell lines. In normal cells, the fluorescence is confined mostly to the nucleus because undamaged DNA cannot migrate. In cells with DNA damage, DNA is denatured by the alkaline solution used for single-strand break detection. The negatively charged DNA fragments are then released from the nucleus and migrate toward the anode. Compared with HBE and HBE-Dp71E cells, HBE-Dp71 AS cells displayed more serious DNA damage (Fig. $2 \mathrm{a}$ and $\mathrm{b}$ ). Then the foci numbers of histone H2AX phosphorylated at serine $139(\gamma-\mathrm{H} 2 \mathrm{AX})$ were examined for detection of DSB (double strand breaks). As indicated in Fig. 2c, clearly more foci of $\gamma-\mathrm{H} 2 \mathrm{AX}$ were detected in HBE-Dp71 AS cells, compared with $\mathrm{HBE}$, and Dp71E cells. Statistical analysis (Fig. 2d) proved that the differences were significant. Alkaline comet assay and $\gamma-\mathrm{H} 2 \mathrm{AX}$ analysis showed enhanced DNA damage induced by $\mathrm{H}_{2} \mathrm{O}_{2}$ in HBE-Dp71 AS cells. 


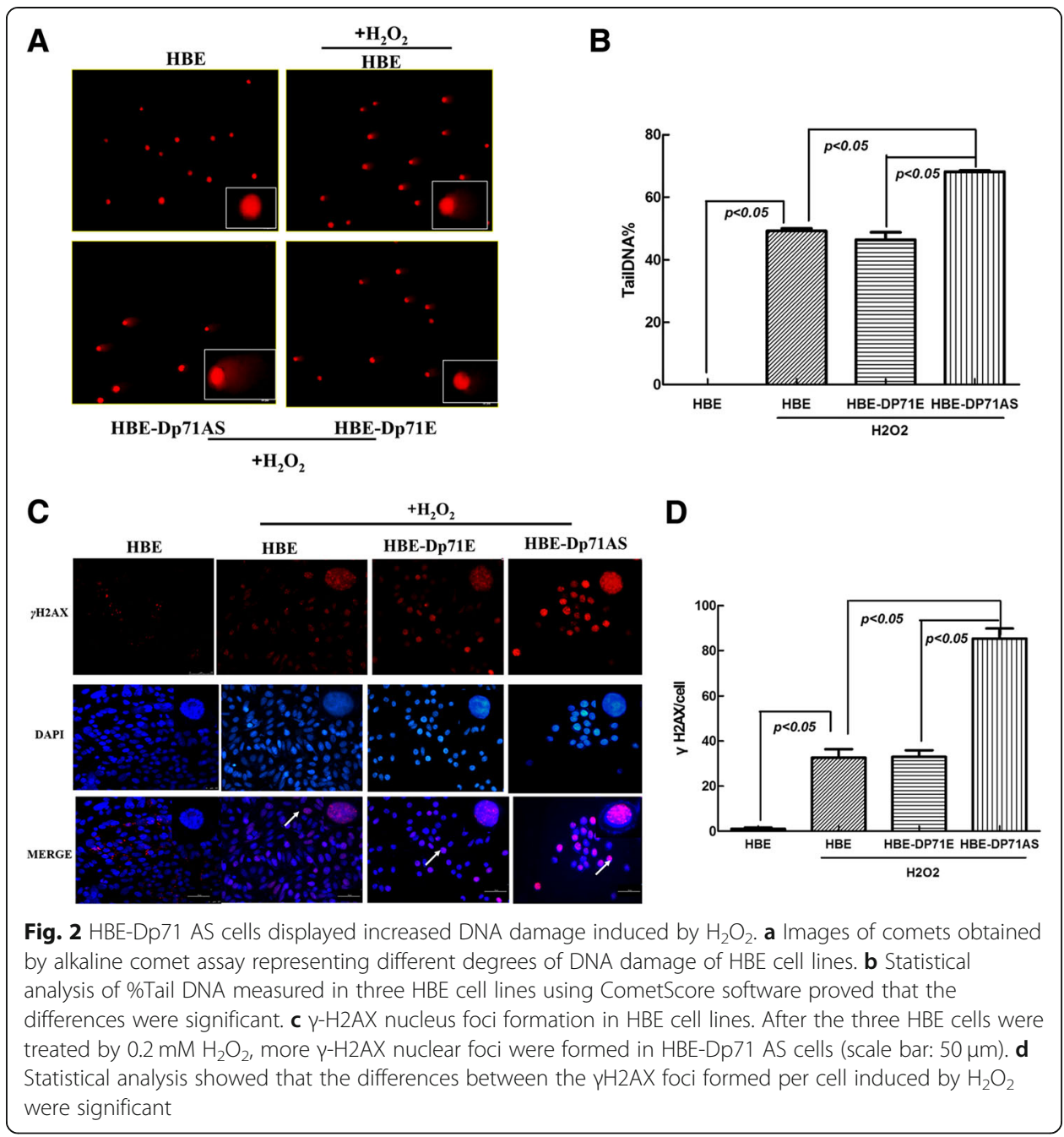

Dp71 knockdown HBE cells displayed increased $\mathrm{H}_{2} \mathrm{O}_{2}$-induced apoptosis via enhanced

\section{caspase 3, caspase 8 and caspase 9 activation}

Cytoskeleton protein is one of the key intracellular components resisting the oxidative stress induced injury which occurs in many circumstances such as ischemia and hypoxia. In order to find out whether the reduction of Dp71 can alter the antioxidant defense capabilities of $\mathrm{HBE}$ cells, apoptosis rates of the three $\mathrm{HBE}$ cell lines induced by $\mathrm{H}_{2} \mathrm{O}_{2}(0.2 \mathrm{mM})$ for $16 \mathrm{~h}$ were analyzed. The apoptosis rates for HBE-Dp71AS, HBE-Dp71E and HBE were $16.00 \pm$ $1.43,7.47 \pm 0.19$ and $7.51 \% \pm 1.27 \%$ respectively after the $\mathrm{H}_{2} \mathrm{O}_{2}$ stimulation. As shown in dot plot images generated by FACS analysis of cells stained with PE Annexin V (Fig. 3a), 8\% more apoptosis was detected in HBE-Dp71AS cells compared with the HBE-Dp71E and HBE cells; the differences were statistically significant (Fig. 3b).

$\mathrm{H}_{2} \mathrm{O}_{2}$ can induce the apoptosis via activation of both the extrinsic death receptor apoptosis pathway and the intrinsic apoptotic pathway. Caspase 9 is an initiator of the intrinsic pathway of apoptosis; caspase 8 is an indispensable enzyme of the extrinsic pathway. The activation of both caspase 8 and 9 will ultimately result in the activation of the effector caspase 3. As shown in Fig. 3c, significant fold change of caspase 3 in HBE-Dp71AS was observed compared with HBE and HBE-Dp71E cells after they had been treated with 


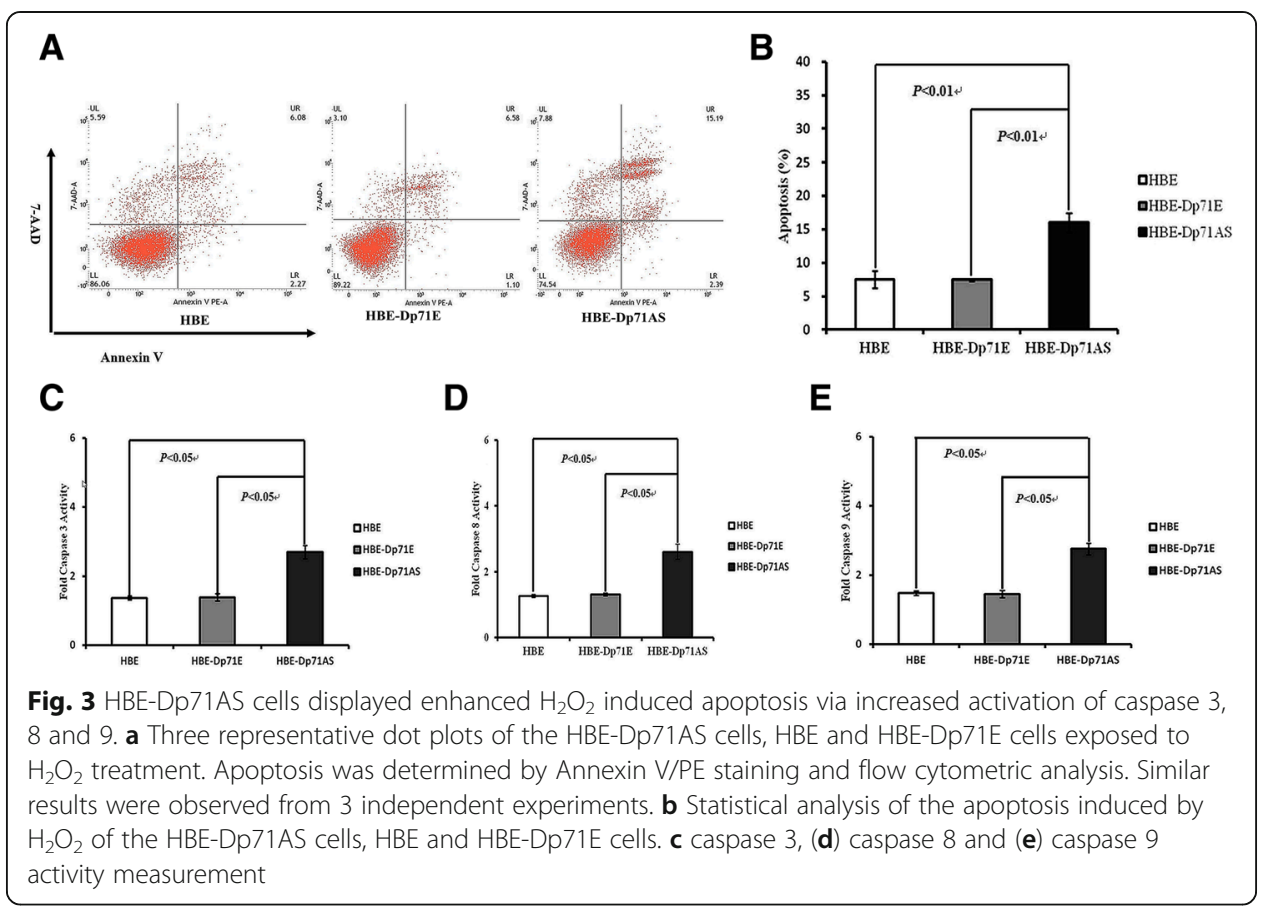

$\mathrm{H}_{2} \mathrm{O}_{2}$. Significant fold changes of caspase 8 and 9 are shown in Fig. 3d and e. To sum up, the ablation of Dp71 increased the $\mathrm{H}_{2} \mathrm{O}_{2}$-induced apoptosis via enhancing the activations of caspase 3,8 and 9 .

\section{Dp71 knockdown HBE cells displayed decreased proliferation rate}

MTT assays were employed to analyze the proliferation of HBE-Dp71AS, HBE-Dp71E and HBE cell groups. Significant growth inhibition was observed at 48 and $72 \mathrm{~h}$ in HBEDp71AS cells (Fig. 4a), while there were no significant differences in cell growth between HBE-Dp71E and HBE cells $(P>0.05)$. Thus the assays indicated that the ablation of Dp71 protein in HBE cells can effectively inhibit their growth.

HBE-Dp71 AS cells displayed reduced clone formation capabilities

The anti-cell proliferation effect of Dp71 was assessed by a clonogenic formation assay. There was a significant reduction in the number and size of foci in HBE-Dp71 AS cells compared with HBE-Dp71E and HBE cells (Fig. 4b). The clone formation efficiency for HBE-Dp71E, HBE and HBE-Dp71AS cells was $85.33 \pm 1.72,88.73 \pm 4.67$ and $67.53 \% \pm$ $3.45 \%$ respectively. The clone formation efficiency for HBE-Dp71AS cells was $20 \%$ less than that in HBE-Dp71E and HBE cells; the differences were statistically significant (Fig. 4c). Together with the results from the MTT assay, it is found that ablation of Dp71 protein can significantly inhibit the growth of HBE cells.

\section{RAD51 interact with Dp71 and Lamin B1 in HBE}

RAD51 is a protein that forms discrete nuclear foci and participates in homologous recombination repair upon DNA damage. Interaction between RAD51 and lamin B1 was verified in previous research $[9,10]$. In our further characterization of Dp71 in HBE cells, it was 
A

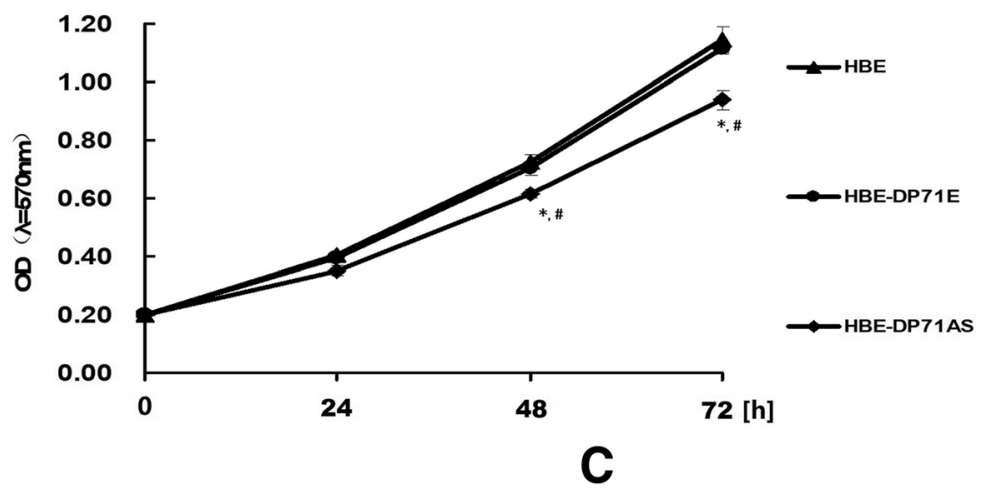

B

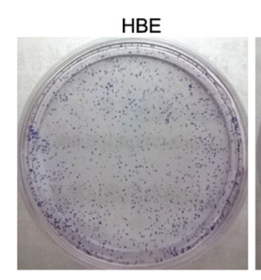
HBE-DP71E

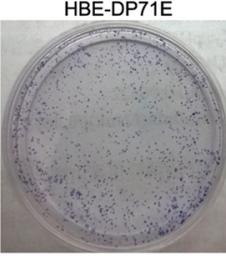

HBE-DP71AS
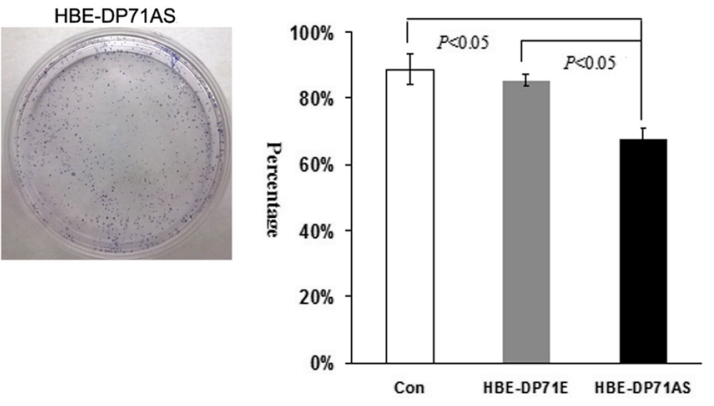

Fig. $4 \mathrm{HBE}-\mathrm{Dp} 71 \mathrm{AS}$ cells displayed inhibited proliferation. a MTT assay was performed to measure the cell growth of HBE-Dp71AS, HBE and HBE-Dp71E cells. The HBE-Dp71AS cells displayed inhibited growth in 24 $\mathrm{h}, 48 \mathrm{~h}$ and $72 \mathrm{~h}$ compared with HBE and HBE-Dp71E cells. The differences in $48 \mathrm{~h}$ and $72 \mathrm{~h}$ are statistically significant, * versus HBE, \# versus HBE-DP71E. b Representative photos of clone formation of HBE-DP71AS, HBE and HBE-Dp71E cells. It is demonstrated that the clonogenic potentials of HBE-Dp71AS were smaller than those of the HBE and HBE-Dp71E cells. Statistical analysis (c) proved that the differences are significant

found that the Dp71 specific antibody pulled down RAD51 successfully, while non-specific IgG failed to pull down RAD51 (Fig. 5a). With RAD51 antibody, Dp71 was successfully precipitated while IgG failed to work (Fig. 5b). Immunofluorescence verified the interaction between RAD51 and Dp71 in the nucleus (Fig. 5d) and cytoplasm (Additional file 1), while the major interaction occurred in the nucleus. Co-Ip results also proved the association between RAD51 and lamin B1. As indicated in Fig. 5b and c, lamin B1 antibody successfully dragged down RAD51, and RAD51 specific antibody precipitated lamin B1. In each assay, however, non-specific IgG failed to precipitate RAD51 and lamin B1. Combined with the proof of RAD51-Dp71 interaction, we proved the existence of Dp71-RAD51-lamin B1 complex in HBE.

Immunoblot was carried out to quantify lamin B1 and RAD51 expression in HBEDp71AS cells. Compared with parental HBE cells and HBE-Dp71E cells, significant reduction of Dp71, lamin B1, RAD51 mRNA (Fig. 5e, f, and g) and protein (Fig. 5h) was observed. The endogenous RAD51 and lamin B1 expression levels were both reduced after the Dp71 expression was knocked down in HBE cells.

\section{Dp71 depletion resulted in decreased FAK, p-FAK and p-AKT}

Dp71 was found associating with most of the $\beta 1$-integrin complex components ( $\beta 1$-integrin, focal adhesion kinase(FAK), $\alpha$-actinin, talin and actin) in PC12 cells [11]. Our 


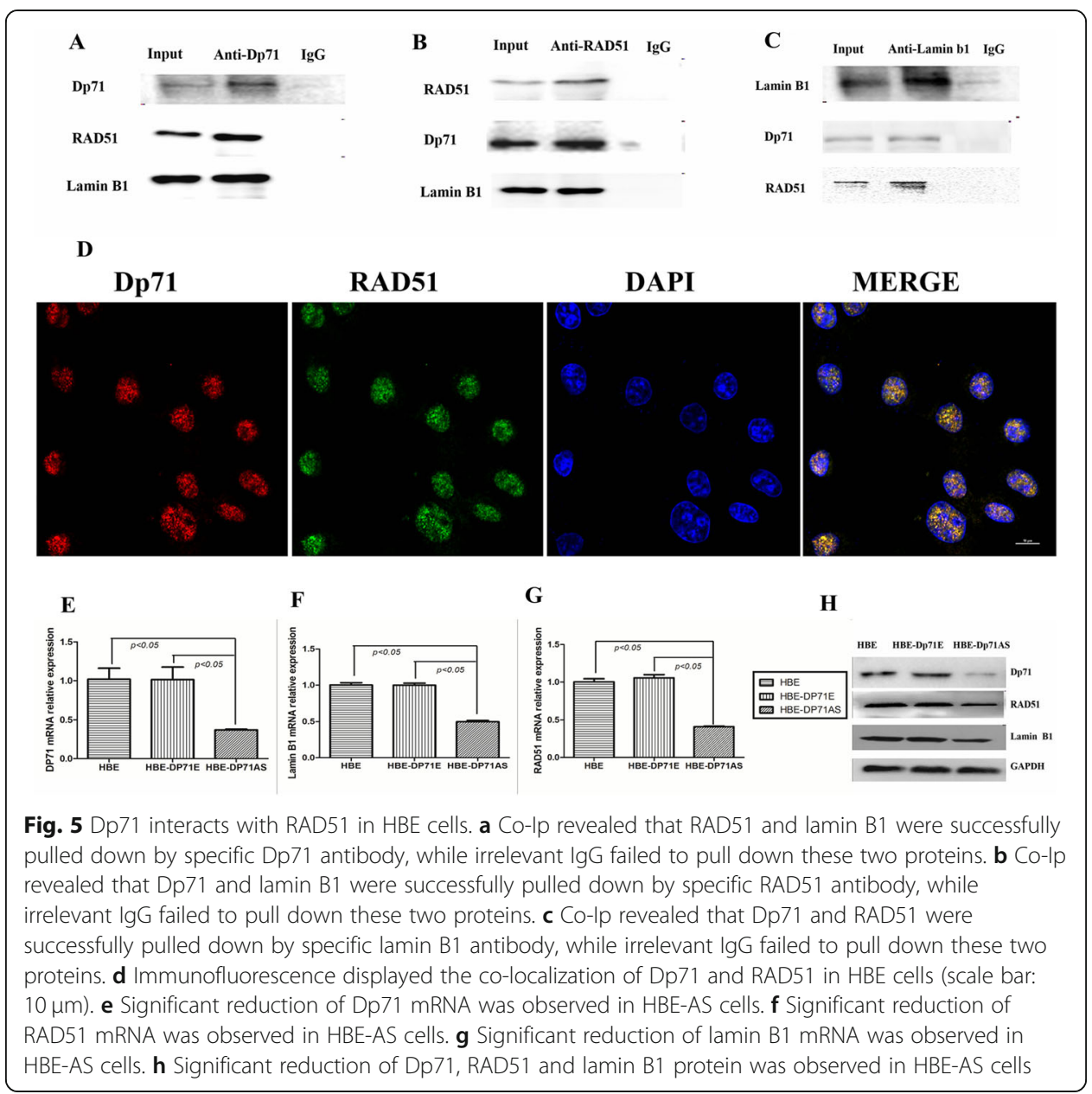

previous publications proved that FAK is an indispensable component of the cytoplasmic DAPCs in HBE. Via two-way precipitation, Fig. $6 a$ and b show that FAK and Dp71 antibody dragged down each other in the immuno-precipitation process while IgG did not precipitate either protein. The co-Ip assay proved that the cytoplasmic association between Dp71 and FAK is a universal phenomenon in different types of cells.

QRT-PCR displayed reduced FAK mRNA in HBE-Dp71AS cells (Fig. 6c, d). Decreased FAK protein and p-FAK expression was also displayed by immuno-blotting in HBE-Dp71AS cells. Although AKT did not display any expression alteration, p-AKT displayed a significant reduction in HBE-Dp71AS cells (Fig. 6e). Knocking down Dp71 in HBE cells resulted in the reduction of cytoplasmic expression of FAK, p-FAK and pAKT.

\section{Discussion}

Being one of the most abundantly expressed dystrophin isoforms, Dp71 is distributed in all tissues except skeletal muscle. The deficiency of Dp71 is involved in mental retardation and retinal dysfunction of DMD patients [2, 12, 13]. For years, PC12 cells have been used as the traditional cell model to study the biological function of Dp71. In our further characterization of this ubiquitously expressed scaffolding protein, Dp71 was found to contain more functional diversity than expected. Being one of the newly 


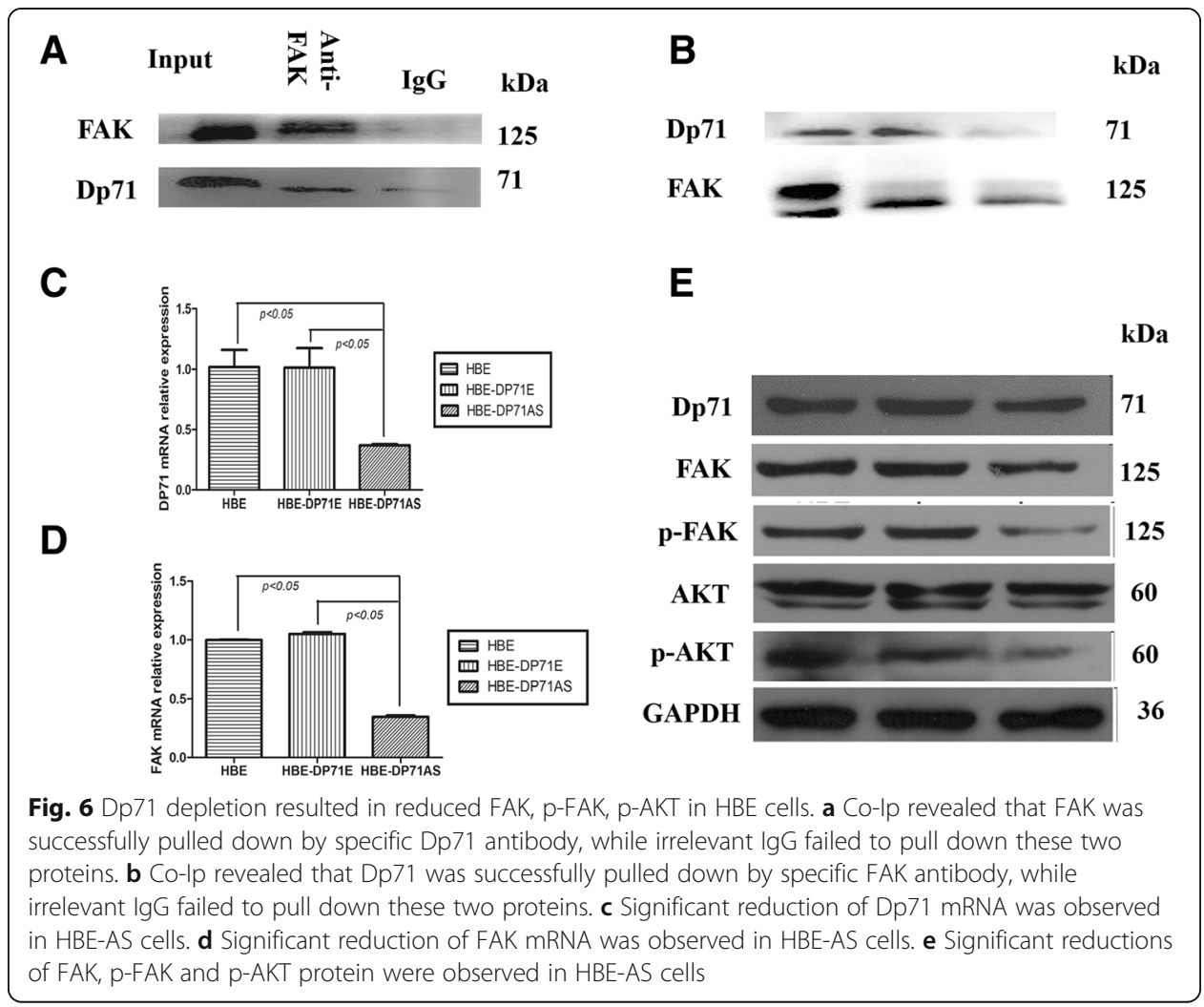

identified tumor suppressive genes in gastric cancer, our preliminary work identified decreased Dp71 in lung cancer as well. As a usual cell model for pulmonary research, we used shRNA to knock down Dp71 and revealed the biological function of HBEDp71AS cells.

After the HBE-Dp71AS cells were stimulated by $\mathrm{H}_{2} \mathrm{O}_{2}$, the alkaline comet assay displayed that tail length and tail moment increased in HBE-Dp71AS cells compared with parental cells and HBE-Dp71E cells. More $\gamma$-H2AX foci were detected in the HBEDp71AS nucleus. That means more single strand break (SSB) and DSB occurred in HBE-Dp71AS cells under oxidative stress. Knocking Dp71 down increased the DNA damage induced by $\mathrm{H}_{2} \mathrm{O}_{2}$.

RAD51 was found to be a new binding partner of Dp71 in our further exploration of HBE-Dp71AS cells. Although cytoplasmic and nuclear interactions between Dp71 and RAD51 were detected in our research, the nuclear Dp71-RAD51 interaction plays a significant role in the increased DNA damage of HBE-Dp71AS cells induced by $\mathrm{H}_{2} \mathrm{O}_{2}$. Co-Ip proved the existence of Dp71-lamin B1-RAD51 protein complex in HBE. RAD51 is proved to be an essential protein for DNA repair by homologous recombination $[14,15]$. Overexpression of Rad51 in different organisms and cell types increased homologous recombination (HR) and increased resistance to DNA damaging agents. Being an associating protein of Dp71, lamin B1 has been found to be a binding partner of RAD51, which promotes DSB repair and cell survival by maintaining the RAD51 protein stability $[9,10]$. In HBE-Dp71AS cells, depletion of Dp71 results in Rad51, lamin B1 mRNA and protein reduction. RAD51 suffers both decreased expression and impaired stability, which leads to 
decreased $\mathrm{HR}$ and increased DNA damage stimulated by $\mathrm{H}_{2} \mathrm{O}_{2}$, and finally results in increased apoptosis.

Via interaction with lamin B1, the nuclear Dp71 is proved to affect the cell growth of PC12 and A549. Decreased lamin B1 expression also slows cell proliferation and induces premature senescence in WI-38 cells $[6,16]$. In HBE-AS cells, significantly reduced lamin B1 explains the phenotypes of growth inhibition. Increased BRCA1 expression caused by decreased lamin B1 reduction leads to the overactivation of caspase 8 and 3, combined with impaired expression and function of RAD51. The apoptosis rate of HBE-Dp71AS cells increased under $\mathrm{H}_{2} \mathrm{O}_{2}$ stimulation.

FAK is a cytosolic non-receptor protein tyrosine kinase that regulates cellular adhesion, motility, proliferation and survival in various types of cells [17-19]. FAK is proved to interact with Dp71 in HBE and PC12. Co-Ip also proved the interaction of FAK with Dp71 in HBE in our current research. Decreased FAK mRNA and protein was observed in HBE-Dp71AS cells. FAK is a novel regulator of DNA damage repair in mutant KRAS NSCLC and its pharmacologic inhibition leads to radiosensitizing effects. Specific targeting of focal adhesion kinase in endothelial cells is sufficient to induce tumor-cell sensitization to DNA-damaging therapies and thus inhibit tumor growth in mice. Although FAK-dependent chemo-sensitivity is proved to be related to DNA-damageinduced NF- $\mathrm{KB}$ activation, the DNA damage response process FAK participates still needs further exploration. FAK-overexpressed (HL-60/FAK) cells were highly resistant to hydrogen peroxide and ionizing radiation (IR)-induced apoptosis [20, 21]. AKT, also known as protein kinase $B$, is a serine/threonine-specific protein kinase responsible for apoptosis, cell proliferation, transcription and cell migration. Accumulating evidence has implicated AKT as a direct participant in the DNA damage response and repair induced by commonly used genotoxic agents. AKT plays an important regulatory role in activating DNA-PKcs and non-homologous end joining (NHEJ) repair [22, 23]. Functioning as an activated downstream target in the FAK pathway, AKT protein expression did not show any change in HBE-AS cells. However, the activated AKT was significantly reduced, which acted together with reduced FAK and RAD51 to attenuate the DNA damage of HBE-Dp71AS cells induced by $\mathrm{H}_{2} \mathrm{O}_{2}$ (Fig. 7). Replication errors after this damage would lead to increased mutations and cancer. The reduced FAK and pAKT can also explain the decreased proliferation of HBE-Dp71AS cells.

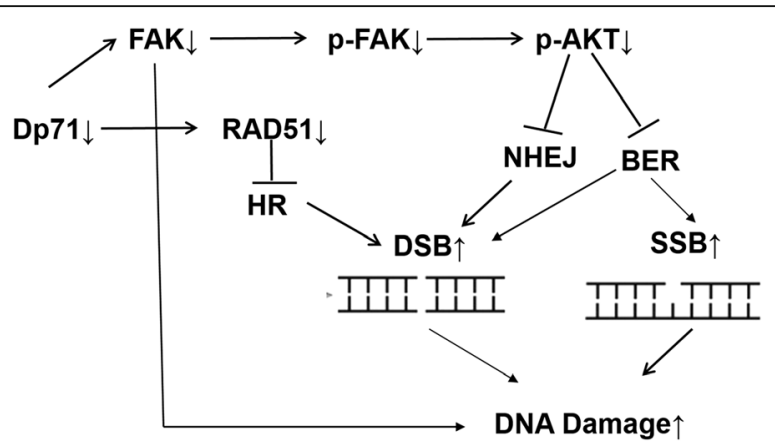

Fig. 7 Schematic map on the mechanism of increased DNA damage induced by $\mathrm{H}_{2} \mathrm{O}_{2}$ in HBE-Dp71AS cells. Combined efforts of reduced RAD51, FAK, and p-Akt in HBE-Dp71AS cells finally result in increased DNA damage stimulated by $\mathrm{H}_{2} \mathrm{O}_{2}$ 
For the first time, it is reported that increased DNA damage occurs in Dp71 depleted cells. With the accumulation of studies showing that many of the human hereditary and non- hereditary cancer predisposition diseases are caused by germ-line mutations in DNA repair genes, the pivotal role that DNA repair plays in the in vivo tumorigenesis process becomes evident. We have proved the in vitro reduction of RAD51 and FAK from the transcription level in HBE-Dp71AS cells. RAD51 and FAK are two important proteins participating in DNA damage repair. Such carcinogens as ROS produced by in vivo metabolism attack DNA and cause a variety of DNA lesions. Unrepaired lesions cause gene mutations and chromosomal damage, which may lead to tumor initiation and progression. However, more experimental evidence is needed to prove the role Dp71 plays in the in vivo tumorigenesis.

Being one of the most ubiquitously expressed isoforms of the dystrophin family, our newly revealed biological traits of HBE-Dp71AS cells suggest a much broader role played by Dp71 in many pathophysiological process, which adds some fresh color to this "old" protein.

\section{Conclusions}

Depletion of Dp71, a widely expressed isoform of dystrophin, displays increased DNA damage, decreased proliferation, and increased apoptosis during oxidative stress via decreasing RAD51, lamin B1, pAKT, FAK and pFAK expression.

\section{Additional file}

Additional file 1: Dp71 interacted with RAD51 in HBE cytoplasm. (JPG $1180 \mathrm{~kb})$

Abbreviations

AKT: Protein kinase B; DMD: Duchenne muscular dystrophy; FAK: Focal adhesion kinase; HBE: Human bronchial epithelium; HR: Homologous recombination; NE: Nuclear envelope; NF-kB: Nuclear factor-kB; QRT-PCR: Quantitative real-time polymerase chain reaction; SSB: Single strand break

Acknowledgements

Not applicable.

\section{Authors' contributions}

SPT and JLX conceived and designed the experiments and wrote the manuscript. SCT, XFX and LX performed the experiments. SZH performed the statistical analysis. All authors read and approved the final manuscript.

\section{Funding}

This study is supported by the National Natural Science Fund of China (Grant No. 81870071), Hunan Natural Science Fund of China (Grant No.2018JJ2628).

Availability of data and materials

Additional information related to this study is available from the author for correspondence upon reasonable request.

Ethics approval and consent to participate

There were no human tissue or animal experiments in this study.

Consent for publication

Not applicable.

Competing interests

The authors declare that they have no competing interests.

Author details

${ }^{1}$ Key Laboratory of Sepsis Translational Medicine of Hunan, Department of Pathophysiology, Xiangya School of Medicine, Central South University, Changsha, Hunan Province 410008, People's Republic of China. ${ }^{2}$ Department of Thoracic Surgery, the Second Xiangya Hospital, Central South University, 139 Ren-min Road, Changsha, Hunan Province 410011, People's Republic of China. ${ }^{3}$ Center of Transplant Surgery, Xiangya Hospital, Central South University, Changsha, Hunan Province 410008, People's Republic of China. ${ }^{4}$ Department of Traditional Chinese Medicine, the Third 
Xiangya Hospital, Central South University, Changsha, Hunan Province, People's Republic of China. ${ }^{5}$ Department of Emergency and Critical Care Medicine, the Third Xiangya Hospital, Central South University, Changsha, Hunan Province, People's Republic of China. ${ }^{6}$ Department of Pathology, Tianjin Medical University Cancer Institute and Hospital, Tianjin 300060, People's Republic of China.

Received: 18 March 2019 Accepted: 5 June 2019

Published online: 17 June 2019

\section{References}

1. Jin H, Tan S, Hermanowski J, Bohm S, Pacheco S, McCauley JM, et al. The dystrotelin, dystrophin and dystrobrevin superfamily: new paralogues and old isoforms. BMC Genomics. 2007;8:19.

2. Austin RC, Howard PL, D'Souza VN, Klamut HJ, Ray PN. Cloning and characterization of alternatively spliced isoforms of Dp71. Hum Mol Genet. 1995:4:1475-83.

3. Bolanos-Jimenez F, Bordais A, Behra M, Strahle U, Sahel J, Rendon A. Dystrophin and Dp71, two products of the DMD gene, show a different pattern of expression during embryonic development in zebrafish. Mech Dev. 2001;102:239-41.

4. Enriquez-Aragon JA, Cerna-Cortes J, Bermudez de Leon M, Garcia-Sierra F, Gonzalez E, Mornet D, Cisneros B. Dystrophin Dp71 in PC12 cell adhesion. Neuroreport. 2005;16:235-8.

5. Aragon J, Romo-Yanez J, Martinez-Herrera A, Ceja V, Rendon A, Montanez C. Characterization of Dp71Delta(78-79), a novel dystrophin mutant that stimulates PC12 cell differentiation. J Neurochem. 2011;119:697-707.

6. Tan S, Tan J, Tan S, Zhao S, Cao X, Chen Z, et al. Decreased Dp71 expression is associated with gastric adenocarcinoma prognosis. Oncotarget. 2016;7:53702-11.

7. Tan S, Zhao S, Chen Z, Ma Q, Wang W, Cheng S, et al. Altered biological properties in Dp71 over-expressing HBE cells. Cell Physiol Biochem. 2017;43:2022-36.

8. Tan S, Tan S, Chen Z, Cheng K, Chen Z, Wang W, et al. Knocking down Dp71 expression in A549 cells reduces its malignancy in vivo and in vitro. Cancer Investig. 2016;34:16-25.

9. Liu NA, Sun J, Kono K, Horikoshi Y, Ikura T, Tong X, et al. Regulation of homologous recombinational repair by Lamin B1 in radiation-induced DNA damage. FASEB J. 2015;29:2514-25.

10. Butin-Israeli V, Adam SA, Jain N, Otte GL, Neems D, Wiesmuller L, et al. Role of Lamin b1 in chromatin instability. Mol Cell Biol. 2015;35:884-98.

11. Cerna J, Osuna-Castro JA, Muniz J, Mornet D, Garcia-Sierra F, Cisneros B. Dystrophin Dp71f associates with components of the beta1-integrin adhesion complex in PC12 cell neurites. Acta Neurol Belg. 2009;109:132-5.

12. Austin RC, Morris GE, Howard PL, Klamut HJ, Ray PN. Expression and synthesis of alternatively spliced variants of Dp71 in adult human brain. Neuromuscul Disord. 2000;10:187-93.

13. Banihani R, Smile S, Yoon G, Dupuis A, Mosleh M, Snider A, McAdam L. Cognitive and neurobehavioral profile in boys with Duchenne muscular dystrophy. J Child Neurol. 2015;30:1472-82.

14. Magwood AC, Malysewich MJ, Cealic I, Mundia MM, Knapp J, Baker MD. Endogenous levels of Rad51 and Brca2 are required for homologous recombination and regulated by homeostatic re-balancing. DNA Repair (Amst). 2013;12:1122-33.

15. Matsunami K, Otsuka H, Xu H, Firdawes S, Yamamoto A, Ishimaru A, Fukuzawa M, Miyagawa S. Molecular cloning of pig Rad51, Rad52, and Rad54 genes, which are involved in homologous recombination machinery. Transplant Proc. 2008;40: 2776-8.

16. Villarreal-Silva M, Centeno-Cruz F, Suarez-Sanchez R, Garrido E, Cisneros B. Knockdown of dystrophin Dp71 impairs PC12 cells cycle: localization in the spindle and cytokinesis structures implies a role for Dp71 in cell division. PLoS One. 2011;6: e23504.

17. Chen JS, Huang XH, Wang Q, Chen XL, Fu XH, Tan HX, Zhang LJ, Li W, Bi J. FAK is involved in invasion and metastasis of hepatocellular carcinoma. Clin Exp Metastasis. 2010;27:71-82.

18. Crosara-Alberto DP, Inoue RY, Costa CR. FAK signalling mediates NF-kappaB activation by mechanical stress in cardiac myocytes. Clin Chim Acta. 2009:403:81-6.

19. Owen KA, Abshire MY, Tilghman RW, Casanova JE, Bouton AH. FAK regulates intestinal epithelial cell survival and proliferation during mucosal wound healing. PLoS One. 2011;6:e23123.

20. Tamagiku Y, Sonoda Y, Kunisawa M, Ichikawa D, Murakami Y, Aizu-Yokota E, Kasahara T. Down-regulation of procaspase8 expression by focal adhesion kinase protects $\mathrm{HL}-60$ cells from TRAlL-induced apoptosis. Biochem Biophys Res Commun. 2004;323:445-52.

21. Huang D, Khoe M, Befekadu M, Chung S, Takata Y, llic D, Bryer-Ash M. Focal adhesion kinase mediates cell survival via NF-kappaB and ERK signaling pathways. Am J Physiol Cell Physiol. 2007;292:C1339-52.

22. Liu Q, Turner KM, Alfred Yung WK, Chen K, Zhang W. Role of AKT signaling in DNA repair and clinical response to cancer therapy. Neuro-Oncology. 2014;16:1313-23.

23. Tavora B, Reynolds LE, Batista S, Demircioglu F, Fernandez I, Lechertier T, Lees DM, Wong PP, Alexopoulou A, Elia G, Clear A, Ledoux A, Hunter J, Perkins N, Gribben JG, Hodivala-Dilke KM. Endothelial-cell FAK targeting sensitizes tumours to DNA-damaging therapy. Nature. 2014;514:112-6.

\section{Publisher's Note}

Springer Nature remains neutral with regard to jurisdictional claims in published maps and institutional affiliations. 